\title{
Precise Detection of Atomicity Violations
}

\author{
Vasco Pessanha, Ricardo J. Dias, and João M. Lourenço \\ Departamento de Informática and CITI \\ Universidade Nova de Lisboa, Portugal \\ $\{v$.pessanha, ricardo.dias\}@campus.fct.unl.pt joao.lourenco@fct.unl.pt
}

\begin{abstract}
Concurrent programs that are free of unsynchronized accesses to shared data may still exhibit unpredictable concurrency errors, called atomicity violations, which include both high-level dataraces and stale-value errors. Atomicity violations occur when programmers make wrong assumptions about the atomicity scope of a code block, incorrectly splitting it in two or more atomic blocks and allow them to be interleaved with other atomic blocks. In this paper we propose a novel static analysis algorithm that works on a dependency graph of program variables and detects both high-level dataraces and stale-value errors. The algorithm was implemented for a Java Bytecode analyzer and its effectiveness was evaluated with well known faulty programs. The results obtained show that our algorithm performs better than previous approaches, achieving higher precision for small and medium sized programs, making it a good basis for a practical tool.
\end{abstract}

\section{Introduction}

The absence or misspecification of the scope of atomic blocks in a concurrent program may trigger atomicity violations and lead to runtime misbehaviors.

Low-level dataraces occur when the program includes unsynchronized accesses to a shared variable, and at least one of those accesses is a write, i.e., it changes the value of the variable. Although low-level dataraces are still a common source of errors and malfunctions in concurrent programs, they have been addressed by others in the past and are out of the scope of this paper. We will consider herein that the concurrent programs under analysis are free from low-level dataraces.

High-level dataraces results from the misspecification of the scope of an atomic block, by splitting it in two or more atomic blocks with other (possibly empty) non-atomic block between them. This anomaly is often referred as a high-level datarace, and is illustrated in Fig. 1(a). A thread uses the method areEqual() to check if the fields ' $a$ ' and ' $b$ ' are equal. This method reads both fields in separate atomic blocks, storing their values in local variables, which are then compared. However, due to an interleaving with another thread running the method setPair (), between lines 12 and 13 the value of the pair may have changed. In this scenario the first thread observes an inconsistent pair, composed by the old value of 'a' and the new value of ' $b$ '. 


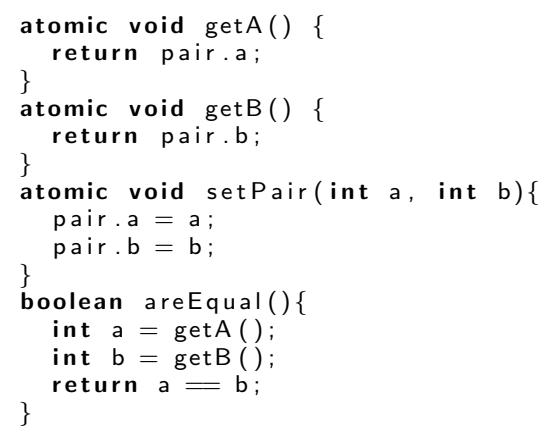

(a) A high-level datarace.

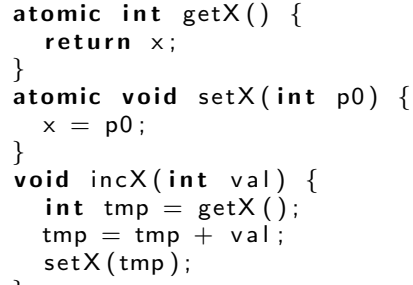

(b) A stale value error.

Fig. 1: Example of atomicity violations.

Figure 1(b) illustrates a stale value error, another source or atomicity violations in concurrent programs. The non-atomic method inc $X()$ is implemented by resorting to two atomic methods, getX() (at line 1) and setX() (at line 4). During the execution of line 9 , if the current thread is suspended and another thread is scheduled to execute setX(), the value of ' $x$ ' changes, and when the execution of the initial thread is resumed it overwrites the value in ' $x$ ' at line 10, causing a lost update. This program fails due to a stale-value error, as at line 8 the value of ' $x$ ' escapes the scope of the atomic method get $X()$ and is reused indirectly (by way of its private copy 'tmp') at line 10 , when updating the value of ' $x$ ' in setX().

In this paper we propose a novel approach for the detection of high-level dataraces and stale-value errors in concurrent programs. As our proposal only depends on the concept of atomic regions and is neutral concerning the mechanisms used for their identification, the atomic regions are not delimited using locks but rather using an @Atomic annotation. Our approach is based on a novel notion of variable dependencies, which we designate as causal dependencies. There is a causal dependency between two variables if the value of one of them influences the writing of the other. We also extended previous work from Artho et al. [2] by reflecting the read/write nature of accesses to shared variables inside atomic regions and additionally use the dependencies information to detect both high-level dataraces and stale-value errors. We formally describe the static analysis algorithms to compute the set of causal dependencies of a program and define safety conditions for both high-level dataraces and stale-value errors.

Our approach can yield both false positives and false negatives. However, the experimental results demonstrate that it still achieves high precision when detecting atomicity violations in well know examples from the literature, suggesting its usefulness for software development tools.

In the next Section of this paper we introduce the previous relevant work on detections of high-level dataraces and stale-value errors; in Section 3 we define a core language and introduce some definitions that support the remainder of the paper, namely Sections 4 and 5, where we propose algorithms for defin- 
ing causal dependencies between variables and for detecting atomicity violations (dataraces). In Section 6 we briefly describe a tool that applies the proposed algorithms with static analysis techniques for Java Bytecode programs, and compare and discuss the results obtained. We terminate in Section 7 with some final concluding remarks.

\section{Background and Related Work}

Several past works address the detection of the same class of atomicity violations in concurrent programs as addressed in this paper.

The work from Artho et al. [2] introduces the concept of view consistency, to detect high-level dataraces. A view of an atomic block is a set containing all the shared variables accessed (both for reading and writing) within that block. The maximal views of a process are those views that are not a subset of any other view. Intuitively, a maximal view defines a set of variables that should always be accessed atomically (inside the same atomic block). A program is free from high-level dataraces if all the views of one thread that are a subset of the maximal views from another thread form an inclusion chain among themselves.

Our work builds on the proposal from Artho et al. [2], but we extend it by incorporating the type of memory access (read or write) into the views, and refine the rules for detecting high-level dataraces to consider this additional information and the information given by the causal dependencies, with considerable positive impact in the precision of the algorithm, as demonstrated in Section 6 .

Praun and Gross [9] introduce method consistency as an extension of view consistency. Based on the intuition that the variables that should be accessed atomically in a given method are all the variables accessed inside a synchronized block, the authors define the concept of method views that relates to Artho et al's maximal views, which aggregates all the shared variables accessed in a method and also differentiates between read and write memory accesses. Similarly to ours, this approach is more precise than Artho et al's because it also detects stale-value errors. Our algorithm however has higher precision than Praun's and give less false positives, as we use maximal views rather than method views.

Wang and Stoller [10] use the concept of thread atomicity to detect and prevent dataraces, where thread atomicity guarantees that all concurrent executions of a set of threads is equivalent to a sequential execution of those threads. In an attempt to reduce the number of false positives yield by [10], Teixeira et al. [7] proposed a variant of this algorithm based in the intuition that the majority of the atomicity violations come from two consecutive atomic blocks that should be merged into a single one. The authors detect dataraces by defining and detecting some anomalous memory access patterns for both high-level dataraces and stale-value errors. Our approach may be seen as a generalization of this concept of memory access patterns, but in our case supported by the notion of causal dependencies between variables, which allow to reduce considerably the number of both false negatives and false positives. 


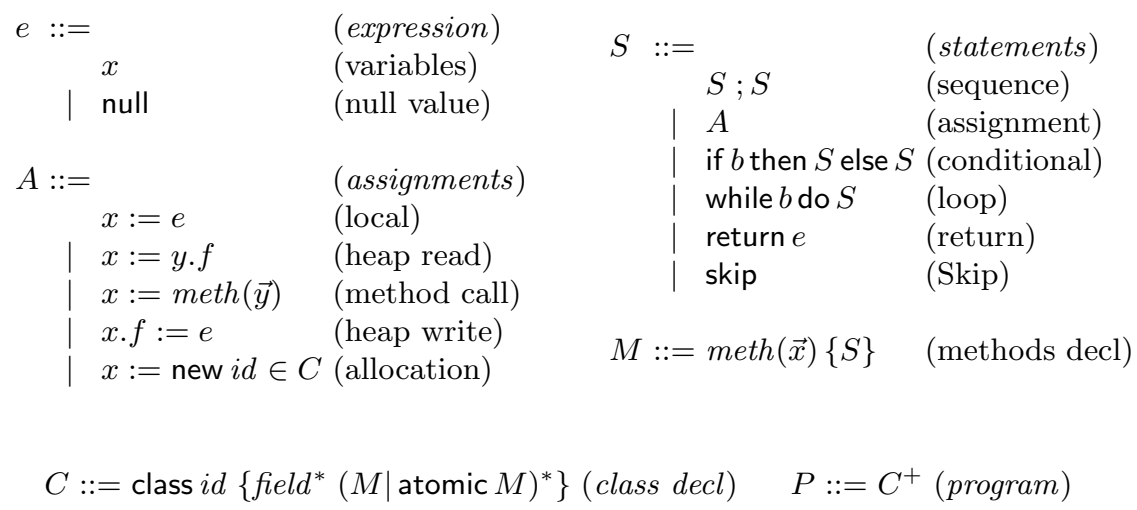

Fig. 2: Core language syntax

\section{Core Language}

We start by defining a core language that captures essential features of a subset of the Java programming language, namely class declaration (class $i d\{\ldots\})$, object creation (new), field dereferencing $(x . f)$, assignment $(x:=e)$, and method invocation $($ meth $(\vec{x}))$. The syntax of the language is defined by the grammar in Fig. 2.

A program in this language is composed by a set of class declarations. Atomic blocks correspond to methods that are declared using the atomic keyword. We require the restriction of not allowing nesting of atomic blocks i.e., we do not allow to call an atomic method inside another atomic method. Variables can hold integers or object references and boolean values are encoded as integers using the value 1 for true and value 0 for false. We also do not support exception handling as normally found in typical object-oriented languages.

We now define some sets that are necessary to the understanding of the following sections:

- Classes: is the set of all class identifiers of all classes declared in the program.

- Fields: is the set of all class fields defined in the program.

- Methods: is the the set of all methods defined in the program.

- Atomics $\subseteq$ Methods: is the subset of methods that were declared as atomic.

We define a local (stack) variable as a pair of the form $(x, m)$ where $x$ is the variable identifier and $m \in$ Methods is the method where this variable is declared. For the sake of simplicity we write the pair $(x, m)$ as only $x$ whenever is not ambiguous to do so. The set of all local variables of a program is denoted as LocalVars.

We define a global variable as an object field and we represent it as the pair $(c, f)$ where $c \in$ Classes represents the class where field $f \in$ Fields is declared. 
The set of all global variables is denoted as GlobalVars. These global variables appear in the code when dereferencing an object reference. For instance, in the statement $x . f:=4$, the expression $x . f$ represents a global variable of the form $(c, f)$ where $c$ is the class of the object reference pointed by local variable $x$. We define a function typeof : LocalVars $\rightarrow$ Classes, which given a local variable returns the class of the object reference that it holds. So, in the example above $c=\operatorname{typeof}(x)$.

Please note that by deciding to represent an access to a field of an object as a pair with the class of the object reference and the field accessed, we are not able to differentiate between different object instances of the same class, and hence we may consider that there is always at most one object instance of each declared class in the program. This allows us to avoid pointer analysis at the cost of losing precision and becoming unsound in some cases but, as the results in Section 6 show, this design choice has proven to be very effective.

Finally we define the set Vars $\equiv$ LocalVars + GlobalVars, which corresponds to all variables used in the program, both local and global variables.

\section{Causal Dependencies}

There is a Causal dependency, which we will designate herein only as dependency, between two program variables (local or global) if the value read from one variable influences the value written into the other. For instance, the following expression

$$
\mathrm{y}:=\mathrm{x}
$$

generates a dependency between variable $x$ and $y$ because the value that is written into variable $y$ was read from variable $x$. As another example, consider the following code:

$$
\text { if }(\mathrm{x}=0)\{\mathrm{y}:=4\}
$$

In this example, the variable $y$ is written only if the condition $x=0$ is true, thus it depends on the current value of variable $x$ and therefore there is also a dependency between variables $x$ and $y$. We represent a dependency between two variables $x$ and $y$ as $x \hookrightarrow y$ where $x \in$ Vars is the variable read and $y \in$ Vars is the variable written.

For each program we can compute a directed graph of causal dependencies. The information provided by this graph plays an important role in finding correlations between variables, which can be used to detect atomicity violations. We can define two kinds of correlations between variables.

Definition 1 (Direct Correlation). There is a direct correlation between a read variable $x$ and a written variable $y$ if there is a path from $x$ to $y$, in a dependency graph $\mathcal{D}$.

Definition 2 (Common Correlation). There is a common correlation between a read variable $x$ and a read variable $y$ if there is a written variable $z$, where $z \neq x$ and $z \neq y$, for which there is a path from $x$ to $z$ and another path from $y$ to $z$, in a dependency graph $\mathcal{D}$. 


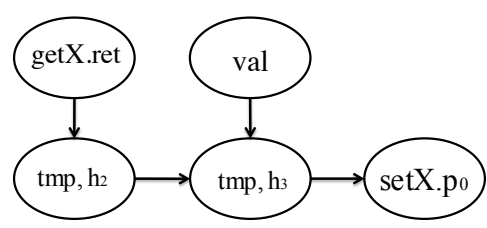

Fig. 3: Dependency graph example

In the following section we describe how to compute the graph of dependencies using symbolic execution.

\subsection{Dependency Analysis}

The construction of the dependency graph is done in two steps. In the first step we only detect data dependencies between variables. In the second step we detect control dependencies between variables. In the end we merge all dependencies in a single graph.

Data Dependencies The accurate detection of data dependencies relies on the precise localisation of where the variables are defined. SSA (Single Static Assignment) [1] could be used, because each variable would only have one definition site, but this only works for local variables, and we still need to track each definition site for global variables. Therefore we did not use SSA as internal representation and we solve the problem by defining a new variable version whenever the variable is updated.

A variable version is defined as a triple of the form $(x, h, m)$ where $x \in$ Vars is a variable (local or global), $h$ is a unique identifier, and $m \in$ Atomics $\cup\{\perp\}$ indicates if this variable is used inside an atomic method or not $(\perp)$. The set of all variable versions is denoted as Versions.

The unique identifier $h$ is a hash value based on the line of code of the respective definition site. If the version of the variable is not known in the current context, as in the case of method arguments, a special hash value is used. We denote this special hash value as $h_{\text {? }}$.

Figure 3 depicts the dependency graph for the method 'incX()' from Fig. 1(b). For the sake of simplicity, we omitted the method $(m)$ part of the version representation. We denote $g e t X$. ret as the return value of method getX(), and setX.pO as the parameter of method setX(int p0). Both the return value and the parameter do not need to have an hash value associated, and thus we omitted it from their representation.

In method incX(int val), the value returned by the method $\operatorname{get} X()$ is written into a temporary variable tmp, which is then incremented using parameter val and is then used as a parameter on the invocation of method setX(int $\mathrm{p} 0$ ).

While analyzing this method, we first start by creating the dependency get $X$. ret $\hookrightarrow\left(t m p, h_{2}\right)$ between the return value of $\operatorname{get} \mathrm{X}()$ method and variable 
tmp with an hash value $h_{2}$. In the next statement variable tmp is redefined with a value resulting from the sum of the previous tmp variable and the val parameter, and hence we create two dependencies $\left(t m p, h_{2}\right) \hookrightarrow\left(t m p, h_{3}\right)$ and val $\hookrightarrow\left(t m p, h_{3}\right)$, where the new version of tmp variable has the hash value $h_{3}$. Finally, we invoke method setX(int $\mathrm{p} 0$ ) with the value of tmp as parameter and therefore we create the dependency $\left(t m p, h_{3}\right) \hookrightarrow \operatorname{set} X . p 0$.

The symbolic execution rules are defined as a transition system $(\langle\mathcal{D}, \mathcal{H}, S\rangle \Longrightarrow$ $\left.\left\langle\mathcal{D}^{\prime}, \mathcal{H}^{\prime}\right\rangle\right)$ over a state composed by a dependency graph $\mathcal{D}$ and a set of versions, denoted has $\mathcal{H} \subseteq$ Versions, which holds the current versions of each program variable. We may find different versions of the same variable, in a single program point, because our analysis over-approximates the run-time state of a program. The rules can be depicted in Figure 4, and we always omit the method $(m)$ parameter from the representation of a variable version.

Function $\operatorname{ver}_{\mathcal{H}}$ is used to retrieve the set of current versions of a variable, and is defined as follows:

Definition 3 (Version Retrieval). Given a set of versions $\mathcal{H}$ and a variable $v \in$ Vars:

$$
\begin{gathered}
\text { ver }: \mathcal{P}(\text { Versions }) \times \text { Vars } \rightarrow \mathcal{P} \text { (Versions) } \\
\operatorname{ver}_{\mathcal{H}}(v) \triangleq \begin{cases}\{(v, h, m) \mid(v, h, m) \in \mathcal{H}\} & \text { if } \exists(v, h, m) \in \mathcal{H} \\
\left\{\left(v, h_{?}, m\right)\right\} & \text { otherwise }\end{cases}
\end{gathered}
$$

If there is not a version for variable $v$, a version with the special hash value $h_{\text {? }}$ is returned.

Every time that a variable is written, it is created a new version for such variable and all other existing current versions are replaced by the new one. We define an helper function $\operatorname{subs}_{\mathcal{H}}$ for this purpose as:

Definition 4 (Version Substitution). Given a set of versions $\mathcal{H}$ and a variable version $(v, h, m) \in$ Versions:

$$
\begin{gathered}
\text { subs }: \mathcal{P}(\text { Versions }) \times \text { Versions } \rightarrow \mathcal{P}(\text { Versions }) \\
\text { subs }_{\mathcal{H}}((v, h, m)) \triangleq\left(\mathcal{H} \backslash\left\{\left(v, h^{\prime}, m^{\prime}\right) \mid\left(v, h^{\prime}, m^{\prime}\right) \in \mathcal{H}\right\}\right) \cup\{(v, h, m)\}
\end{gathered}
$$

Each hash value is generated using function nhash, which given a statement $S$, generates a new, and unique, hash value based of the line number of statement $S$. This function is deterministic in the sense that for any statement $S$ the same hash value is always returned.

At the beginning of the analysis, the sets $\mathcal{D}$ and $\mathcal{H}$ are empty. We represent meth. $p_{i}$ as the parameters of methods, and meth.ret as the return value of a method. We denote as retVar the return value of the method that is being analysed when evaluating the RETURN statement.

All assignment operations, namely Assign, HeAP READ, and Heap Write, create dependencies between all versions of the variables used in the right side of 


$$
\begin{aligned}
& \frac{\left\langle\mathcal{D}, \mathcal{H}, S_{1}\right\rangle \Longrightarrow\left\langle\mathcal{D}^{\prime}, \mathcal{H}^{\prime}\right\rangle \quad\left\langle\mathcal{D}^{\prime}, \mathcal{H}^{\prime}, S_{2}\right\rangle \Longrightarrow\left\langle\mathcal{D}^{\prime \prime}, \mathcal{H}^{\prime \prime}\right\rangle}{\left\langle\mathcal{D}, \mathcal{H}, S_{1} ; S_{2}\right\rangle \Longrightarrow\left\langle\mathcal{D}^{\prime \prime}, \mathcal{H}^{\prime \prime}\right\rangle}(\mathrm{SEQ}) \\
& h=\operatorname{nhash}(x:=y) \\
& \frac{\mathcal{H}^{\prime}=\operatorname{subs}_{\mathcal{H}}((x, h)) \quad \mathcal{D}^{\prime}=\mathcal{D} \cup\left\{v \hookrightarrow(x, h) \mid v \in \operatorname{ver}_{\mathcal{H}}(y)\right\}}{\langle\mathcal{D}, \mathcal{H}, x:=y\rangle \Longrightarrow\left\langle\mathcal{D}^{\prime}, \mathcal{H}^{\prime}\right\rangle}(\text { Assign }) \\
& c=\operatorname{typeof}(y) \quad h=\operatorname{nhash}(x:=y . f) \quad \mathcal{H}^{\prime}=\operatorname{subs}_{\mathcal{H}}((x, h)) \\
& \mathcal{D}^{\prime}=\mathcal{D} \cup\left\{v \hookrightarrow(x, h) \mid v \in \operatorname{ver}_{\mathcal{H}}((c, f))\right\} \\
& \langle\mathcal{D}, \mathcal{H}, x:=y \cdot f\rangle \Longrightarrow\left\langle\mathcal{D}^{\prime}, \mathcal{H}^{\prime}\right\rangle \\
& c=\operatorname{typeof}(x) \quad h=\operatorname{nhash}(x . f:=y) \quad \mathcal{H}^{\prime}=\operatorname{subs}_{\mathcal{H}}(((c, f), h)) \\
& \mathcal{D}^{\prime}=\mathcal{D} \cup\left\{v \hookrightarrow((c, f), h) \mid v \in \operatorname{ver}_{\mathcal{H}}(y)\right\} \\
& \langle\mathcal{D}, \mathcal{H}, x . f:=y\rangle \Longrightarrow\left\langle\mathcal{D}^{\prime}, \mathcal{H}^{\prime}\right\rangle \\
& \frac{h=\operatorname{nhash}(x:=\operatorname{new} C()) \quad \mathcal{H}^{\prime}=\operatorname{subs}_{\mathcal{H}}((x, h))}{\langle\mathcal{D}, \mathcal{H}, x:=\operatorname{new} C()\rangle \Longrightarrow\left\langle\mathcal{D}, \mathcal{H}^{\prime}\right\rangle}(\text { Allocation }) \\
& h=\operatorname{nhash}(x:=\operatorname{meth}(\vec{y})) \quad \operatorname{spec}(\text { meth })=\left\langle\mathcal{D}_{f}, \mathcal{H}_{f}\right\rangle \quad \mathcal{D}^{\prime}=\mathcal{D}_{f} \cup \mathcal{D} \\
& \mathcal{D}^{\prime \prime}=\mathcal{D}^{\prime} \cup\left\{v_{i} \hookrightarrow \text { meth. } p_{i} \mid y_{i} \in \vec{y} \wedge v_{i} \in \operatorname{ver}_{\mathcal{H}}\left(y_{i}\right)\right\} \cup\{\text { meth. ret } \hookrightarrow(x, h)\} \\
& \mathcal{H}^{\prime}=\left\{(v, h) \mid(v, h) \in \mathcal{H} \wedge\left(\left(v, h_{?}\right) \in \mathcal{H}_{f} \vee(v, h) \notin \mathcal{H}_{f}\right)\right\} \\
& \mathcal{H}^{\prime \prime}=\left\{(v, h) \mid(v, h) \in \mathcal{H}_{f} \wedge h \neq h_{?}\right\} \\
& \langle\mathcal{D}, \mathcal{H}, x:=\operatorname{meth}(\vec{y})\rangle \Longrightarrow\left\langle\mathcal{D}^{\prime \prime}, \mathcal{H}^{\prime} \cup \mathcal{H}^{\prime \prime}\right\rangle \\
& \left\langle\mathcal{D}, \mathcal{H}, S_{1}\right\rangle \Longrightarrow\left\langle\mathcal{D}^{\prime}, \mathcal{H}^{\prime}\right\rangle \quad\left\langle\mathcal{D}, \mathcal{H}, S_{2}\right\rangle \Longrightarrow\left\langle\mathcal{D}^{\prime \prime}, \mathcal{H}^{\prime \prime}\right\rangle \\
& \mathcal{H}^{\prime \prime \prime}=\mathcal{H}^{\prime} \cup \mathcal{H}^{\prime \prime} \cup\left\{\left(v, h_{?}\right) \mid\left(v, h_{1}\right) \in \mathcal{H}^{\prime} \wedge\left(v, h_{2}\right) \notin \mathcal{H}^{\prime \prime}\right\} \\
& \cup\left\{\left(v, h_{?}\right) \mid\left(v, h_{1}\right) \in \mathcal{H}^{\prime \prime} \wedge\left(v, h_{2}\right) \notin \mathcal{H}^{\prime}\right\} \\
& \left\langle\mathcal{D}, \mathcal{H}, \text { if } b \text { then } S_{1} \text { else } S_{2}\right\rangle \Longrightarrow\left\langle\mathcal{D}^{\prime} \cup \mathcal{D}^{\prime \prime}, \mathcal{H}^{\prime \prime \prime}\right\rangle \\
& \langle\mathcal{D}, \mathcal{H}, S\rangle \Longrightarrow\left\langle\mathcal{D}^{\prime}, \mathcal{H}^{\prime}\right\rangle \quad \mathcal{H}^{\prime \prime}=\mathcal{H} \cup \mathcal{H}^{\prime} \cup\left\{\left(v, h_{?}\right) \mid\left(v, h_{1}\right) \in \mathcal{H} \wedge\left(v, h_{2}\right) \notin \mathcal{H}^{\prime}\right\} \\
& \cup\left\{\left(v, h_{?}\right) \mid\left(v, h_{1}\right) \in \mathcal{H}^{\prime} \wedge\left(v, h_{2}\right) \notin \mathcal{H}\right\} \\
& \langle\mathcal{D}, \mathcal{H} \text {, while } b \text { do } S\rangle \Longrightarrow\left\langle\mathcal{D} \cup \mathcal{D}^{\prime}, \mathcal{H}^{\prime \prime}\right\rangle \\
& \frac{\mathcal{D}^{\prime}=\mathcal{D} \cup\left\{v \hookrightarrow \operatorname{retVar} \mid v \in \operatorname{ver}_{\mathcal{H}}(x)\right\}}{\langle\mathcal{D}, \mathcal{H}, \text { return } x\rangle \Longrightarrow\left\langle\mathcal{D}^{\prime}, \mathcal{H}\right\rangle}(\text { Return }) \quad \frac{}{\langle\mathcal{D}, \mathcal{H}, \text { skip }\rangle \Longrightarrow\langle\mathcal{D}, \mathcal{H}\rangle}(\text { SKIP })
\end{aligned}
$$

Fig. 4: Symbolic execution rules of data dependencies analysis

the assignment and the new version of the assigned variable. The newly generated version is then used to replace all existing versions of the same variable.

In the rule MEтH CALL, the function spec returns the result, denoted as $\left\langle\mathcal{D}_{p}, \mathcal{H}_{p}\right\rangle$, of the analysis of method meth. The dependencies in $\mathcal{D}_{p}$ are merged with the current dependencies and we create a dependency between each value that is passed as an argument to meth and the respective declared parameter meth. $p_{i}$. We also need to update the variables' versions that are generated inside the method. If a variable was redefined $\left(h \neq h_{?}\right)$ inside meth then we replace the existing versions with the new version, otherwise we keep the current versions. Finally, we add one more dependency between the return value of method meth and the assigned value. 
In the rule ConditionaL, the dependencies are generated in both branches and are merged with the initial $\mathcal{D}$. We also generate the versions for each branch, and if a variable $x$ has a version $h \neq h_{\text {? }}$ in one branch but there is not any version for the same variable in the other branch, then we generate a special version $h_{\text {? }}$ for variable $x$ and we join it to all the other versions. The intuition behind this operation is that if a variable is written only in one of the branches then we also need to add the case that the variable might not have been written. The rule LoOP is similar to the CONDITIONAL rule. The remaining rules should be self-explanatory.

After analyzing all methods of the program we get a dependency graph for the whole program, based on data-flow information. Next, we have to add the remaining dependencies based on the control flow information.

Control Dependencies If an assignment or return statement is guarded by some condition then that assignment or return statement depends on the variables that compose the condition. This situation may occur with every conditional statement such as an if then else, or a while loop.

The analysis of control dependencies traverses the control flow graph and keeps the set of variables that the assignments may depend on. When an assignment or return statement is found we create a dependency between the current variables, that it may depend on, and the respective assigned variable.

The symbolic execution rules are shown in Figure 5 as a transition system $\left(\langle\mathcal{I} \mathcal{S}, \mathcal{D}, S\rangle \Longrightarrow\left\langle\mathcal{I S}^{\prime}, \mathcal{D}^{\prime}\right\rangle\right)$. The state is composed by a set of conditional variables $\mathcal{I} \mathcal{S} \subseteq$ Versions, which correspond to the variable versions that the current statement depends on, and a dependency graph $\mathcal{D}$. In the beginning of the analysis the dependency graph is empty, and the set of conditional variables has the union of all conditional variables that are present at all calling contexts of the method that is going to be analyzed. For instance, given the program methods $m_{1}, m_{2}$ and $m_{3}$ where method $m_{1}$ calls method $m_{2}$ with the current conditional variables set $\mathcal{I S}=\left\{c_{1}, c_{2}\right\}$, and $m_{3}$ calls method $m_{2}$ with the current conditional variables set $\mathcal{I S}=\left\{c_{3}, c_{4}\right\}$, then the initial set of conditional variables when analyzing method $m_{2}$ is $\mathcal{I S}=\left\{c_{1}, c_{2}, c_{3}, c_{4}\right\}$.

In the end of this analysis the resulting graph of dependencies is merged with the one that resulted from the data dependencies analysis, described in the previous section, thus forming the complete graph of causal dependencies.

For every kind of assignment we create a dependency between the current conditional variables and the assigned variable. This situation may occur in the rules Assign, Heap Read, Heap Write, Allocation and Meth Call. In the case of a return statement, as in rule RETURN, we create a dependency with the special variable retVar.

In the rules Conditional and Loop, we analyze each branch with a new set of conditional variables composed by the current conditional variables plus the variable of the condition. The variable is actually a variable version with an unique hash value. When we exit the scope of the condition we remove the 


$$
\begin{aligned}
& \frac{\left\langle\mathcal{I S}, \mathcal{D}, S_{1}\right\rangle \Longrightarrow\left\langle\mathcal{I} \mathcal{S}^{\prime}, \mathcal{D}^{\prime}\right\rangle \quad\left\langle\mathcal{I S}^{\prime}, \mathcal{D}^{\prime}, S_{2}\right\rangle \Longrightarrow\left\langle\mathcal{I} \mathcal{S}^{\prime \prime}, \mathcal{D}^{\prime \prime}\right\rangle}{\left\langle\mathcal{I} \mathcal{S}, \mathcal{D}, S_{1} ; S_{2}\right\rangle \Longrightarrow\left\langle\mathcal{I} \mathcal{S}^{\prime \prime}, \mathcal{D}^{\prime \prime}\right\rangle}(\mathrm{SEQ}) \\
& \frac{h=\operatorname{nhash}(x:=y) \quad \mathcal{D}^{\prime}=\mathcal{D} \cup\{v \hookrightarrow(x, h) \mid v \in \mathcal{I S}\}}{\langle\mathcal{I} \mathcal{S}, \mathcal{D}, x:=y\rangle \Longrightarrow\left\langle\mathcal{I} \mathcal{S}, \mathcal{D}^{\prime}\right\rangle}(\text { Assign }) \\
& \frac{h=\operatorname{nhash}(x:=y \cdot f) \quad \mathcal{D}^{\prime}=\mathcal{D} \cup\{v \hookrightarrow(x, h) \mid v \in \mathcal{I} \mathcal{S}\}}{\langle\mathcal{I} \mathcal{S}, \mathcal{D}, x:=y \cdot f\rangle \Longrightarrow\left\langle\mathcal{I} \mathcal{S}, \mathcal{D}^{\prime}\right\rangle}(\text { HeAP READ })
\end{aligned}
$$

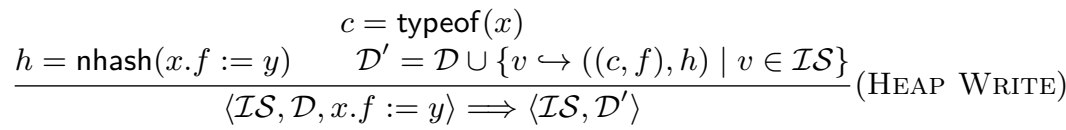

$$
\begin{aligned}
& \frac{h=\operatorname{nhash}(x:=\operatorname{new} C()) \quad \mathcal{D}^{\prime}=\mathcal{D} \cup\{v \hookrightarrow(x, h) \mid v \in \mathcal{I} \mathcal{S}\}}{\langle\mathcal{I} \mathcal{S}, \mathcal{D}, x:=\operatorname{new} C()\rangle \Longrightarrow\left\langle\mathcal{I} \mathcal{S}, \mathcal{D}^{\prime}\right\rangle} \text { (Allocation) } \\
& h=\operatorname{nhash}(x:=\operatorname{meth}(\vec{y})) \\
& \frac{\operatorname{spec}(\text { meth })=\left\langle\mathcal{I} \mathcal{S}_{f}, \mathcal{D}_{f}\right\rangle \quad \mathcal{D}^{\prime}=\mathcal{D} \cup \mathcal{D}_{f} \cup\{v \hookrightarrow(x, h) \mid v \in \mathcal{I} \mathcal{S}\}}{\langle\mathcal{I} \mathcal{S}, \mathcal{D}, x:=\operatorname{meth}(\vec{y})\rangle \Longrightarrow\left\langle\mathcal{I} \mathcal{S}, \mathcal{D}^{\prime}\right\rangle}(\text { Meth CALL) } \\
& \mathcal{I S}^{\prime}=\mathcal{I S} \cup\{b\} \\
& \frac{\left\langle\mathcal{I} \mathcal{S}^{\prime}, \mathcal{D}, S_{1}\right\rangle \Longrightarrow\left\langle\mathcal{I} \mathcal{S}^{\prime}, \mathcal{D}^{\prime}\right\rangle \quad\left\langle\mathcal{I} \mathcal{S}^{\prime}, \mathcal{D}, S_{2}\right\rangle \Longrightarrow\left\langle\mathcal{I} \mathcal{S}^{\prime}, \mathcal{D}^{\prime \prime}\right\rangle}{\left\langle\mathcal{I} \mathcal{S}, \mathcal{D} \text {, if } b \text { then } S_{1} \text { else } S_{2}\right\rangle \Longrightarrow\left\langle\mathcal{I} \mathcal{S}, \mathcal{D}^{\prime} \cup \mathcal{D}^{\prime \prime}\right\rangle}(\text { Conditional }) \\
& \frac{\mathcal{I S}^{\prime}=\mathcal{I S} \cup\{b\} \quad\left\langle\mathcal{I} \mathcal{S}^{\prime}, \mathcal{D}, S\right\rangle \Longrightarrow\left\langle\mathcal{I S}^{\prime}, \mathcal{D}^{\prime}\right\rangle}{\langle\mathcal{I} \mathcal{S}, \mathcal{D} \text {, while } b \text { do } S\rangle \Longrightarrow\left\langle\mathcal{I} \mathcal{S}, \mathcal{D} \cup \mathcal{D}^{\prime}\right\rangle}(\mathrm{LoOP}) \\
& \frac{\mathcal{D}^{\prime}=\mathcal{D} \cup\{v \hookrightarrow \text { retVar } \mid v \in \mathcal{I S}\}}{\langle\mathcal{I} \mathcal{S}, \mathcal{D}, \text { return } x\rangle \Longrightarrow\left\langle\mathcal{I S}, \mathcal{D}^{\prime}\right\rangle}(\text { RETURN }) \quad \frac{}{\langle\mathcal{I S}, \mathcal{D}, \text { skip }\rangle \Longrightarrow\langle\mathcal{I} \mathcal{S}, \mathcal{D}\rangle}(\mathrm{SKIP})
\end{aligned}
$$

Fig. 5: Symbolic execution rules of control dependencies analysis

condition variable and proceed with the analysis. The remaining rules are selfexplanatory.

The result of these two analysis generate the graph of causal dependencies that is used to detect the existence of atomicity violations in a concurrent program, as we will show in the following sections.

\section{Atomicity Violations}

The purpose of our work is to detect two kinds of atomicity errors, the highlevel datarace and the stale-value error, that may occur during the execution of concurrent programs that use atomic blocks to guarantee mutual exclusion in the access to shared data.

The definition of both errors assume that the concurrent program has no low-level dataraces, meaning that all accesses to shared variables are done inside atomic blocks. 


\subsection{High Level Dataraces}

A view, as described by Artho et al. in [2], expresses what variables are accessed inside a given atomic code block. We extend this definition by also keeping the kind of access (read or write) that was made for each variable in the view.

Please note that a view only stores global variables. Local variables are not shared between threads and thus do not require synchronized accesses.

We denote as Accesses the set of memory accesses made inside an atomic block. An access $a \in$ Accesses is a pair of the form $(\alpha, v)$ where $\alpha \in\{r, w\}$ represents the kind of access ( $r$-read or $w$-write) and $v \in$ GlobalVars is a global variable $^{1}$. A view is a subset of Accesses and the set of all views in a program is denoted as Views. A view is always associated with one atomic method, and we define the bijective function $\Gamma$ that given a view returns the associated atomic method as:

$$
\Gamma: \text { Views } \rightarrow \text { Atomics }
$$

The inverse function, denoted as $\Gamma^{-1}$, returns the view associated with a given atomic method. The set of generated views of a process $p$, denoted as $V(p)$, corresponds to the atomic blocks executed by one process, and is defined as:

$$
v \in V(p) \Leftrightarrow m=\Gamma(v) \wedge \operatorname{executes}(p, m)
$$

The predicate executes asserts if a method $m$ may be executed by process $p$, and is defined by an auxiliary static analysis that computes the set of processes and the atomic methods that are called in each process.

We can refine the previous definition of $V(p)$ with a parameter $\alpha$, where $\alpha \in\{r, w\}$, to get only the views of a process with read $\left(V_{r}\right)$ or write accesses $\left(V_{w}\right)$.

\section{Definition 5 (Procedure Views).}

$$
V_{\alpha}(p)=\left\{v_{2} \mid v_{1} \in V(p) \wedge v_{2}=\left\{(\alpha, x) \mid(\alpha, x) \in v_{1}\right\}\right\} \quad \text { where } \alpha \in\{r, w\}
$$

We defined a static analysis to compute a view of an atomic method. Every time a global variable is read or written, the corresponding read or write access is created and added to the view. The view resulting from a method call is merged with the current view that is being computed. In the case of conditional and loop statements we perform an over-approximation union of the views of each branch. In the end of the analysis we have the set of views corresponding to the atomic methods present in the program code.

The maximal views of a process, denoted as $M_{\alpha}$, are all the views of the process that are not a subset of any other view in that same process. A maximal view is defined as follows:

${ }^{1}$ Please remember that global variables are represented as a pair with a class identifier and the field accessed. 
Definition 6 (Maximal Views). Given a process $p$, a maximal view $v_{m}$ is defined as:

$v_{m} \in M_{\alpha}(p) \Leftrightarrow v_{m} \in V_{\alpha}(p) \wedge\left(\forall v \in V_{\alpha}(p): v_{m} \subseteq v \Rightarrow v=v_{m}\right) \quad$ where $\alpha \in\{r, w\}$

Each maximal view represent the set of variables that should be accessed atomically, i.e., should always be accessed in the same atomic block.

Given a set of views of a process $p$ and a maximal view $v_{m}$ of another process, we define the read or write overlapping views of process $p$ with view $v_{m}$ as all the non empty intersection views between $v_{m}$ and the views of process $p$.

Definition 7 (Overlapping Views). Given a process $p$ and maximal view $v_{m}$ :

$$
\operatorname{overlap}_{\alpha}\left(p, v_{m}\right) \triangleq\left\{v_{m} \cap v \mid v \in V_{\alpha}(p) \wedge v_{m} \cap v \neq \emptyset\right\} \quad \text { where } \alpha \in\{r, w\}
$$

The notion of compatibility between a process $p$ and a view $v_{m}$, defined in [2], states that a process $p$ and a view $v_{m}$ are compatible if all their overlapping views form a chain. We extended this definition with the information given by the causal dependencies graph, and we additionally require that, even if the read overlapping views do not form a chain, there may not exist a common correlation (Definition 2) between the variables in the read overlapping views.

Definition 8 (Process Compatibility). Given a process $p$ and maximal view $v_{m}$ :

$$
\begin{array}{r}
\operatorname{comp}_{w}\left(p, v_{m}\right) \Leftrightarrow \forall v_{1}, v_{2} \in \operatorname{overlap}_{w}\left(p, v_{m}\right): v_{1} \subseteq v_{2} \vee v_{2} \subseteq v_{1} \\
\operatorname{comp}_{r}\left(p, v_{m}\right) \Leftrightarrow \forall v_{1}, v_{2} \in \operatorname{overlap}_{r}\left(p, v_{m}\right): v_{1} \subseteq v_{2} \vee v_{2} \subseteq v_{1} \\
\vee \neg \text { CommonCorrelation }\left(\mathrm{v}_{1}, \mathrm{v}_{2}\right)
\end{array}
$$

The intuition behind this additional condition is that, even if two shared variables that belong to a maximal view were read in different atomic blocks, only if both variables are used in a common write operation we will consider that there is an incompatibility.

We can now define the view consistency safety property in terms of the compatibility between all pairs of processes of a program. A process may only have views that are compatible with all maximal views of another process. A program is free from high-level dataraces if the following condition holds:

\section{Definition 9 (View Consistency).}

$$
\forall p_{1}, p_{2} \in \mathcal{P}_{\mathcal{S}}, m_{r} \in M_{r}\left(p_{1}\right), m_{w} \in M_{w}\left(p_{1}\right): \operatorname{comp}_{w}\left(p_{2}, m_{r}\right) \wedge \operatorname{comp}_{r}\left(p_{2}, m_{w}\right)
$$

where $\mathcal{P}_{\mathcal{S}}$ is the set of processes. 


\subsection{Stale-Value Error}

Stale-value errors are a class of atomicity violations that are not detected by the view consistency property. Our approach to detect these kind of errors uses the graph of causal dependencies to detect values that escape the scope of an atomic block (e.g., by assigning a shared variable to a local variable) and are later used inside another atomic block (e.g., by assigning the previous local variable to a shared variable).

First we define the set IVersions $\subseteq$ Versions, which stores all global variable versions that were accessed inside an atomic block. Each variable version has a parameter $m$ that indicates in which atomic method it was defined, or has the value $\perp$ if it was not used inside an atomic method.

Definition 10 (Atomic Variable Version). A global variable version $(x, h, m)$ is an atomic variable if:

$$
(x, h, m) \in \text { IVersions } \Leftrightarrow(x, h, m) \in \text { Versions } \wedge x \in \text { GlobalVars } \wedge m \neq \perp
$$

Now we define a new graph, denoted as $\mathcal{D}_{\mathcal{V}}$, which represent the dependencies between views. A labeled edge of this graph $\mathcal{D}_{\mathcal{V}}$ is represented as $\left(m_{1}, x, m_{2}\right)$ where $m_{1}, m_{2} \in$ Atomics and $x \in$ GlobalVars, and can be interpreted as atomic method $m_{2}$ depends on atomic method $m_{1}$ through global variable $x$. Intuitively, this means that the value of variable $x$ exited the scope of method $m_{1}$ and entered the scope of method $m_{2}$, and while it was out of the atomic scopes, it might have become outdated.

Each edge $\left(m_{1}, x_{1}, m_{2}\right)$ of a view dependency graph $\mathcal{D}_{\mathcal{V}}$, is created when, given two version variables $a_{1}=\left(x_{1}, h_{1}, m_{1}\right) \in$ IVersions and $a_{2}=\left(x_{2}, h_{2}, m_{2}\right) \in$ IVersions, and a causal dependency graph $\mathcal{D}$, the following conditions hold:

$$
\begin{aligned}
&\left(\text { DirectCorrelation }\left(\mathcal{D}, a_{1}, a_{2}\right) \wedge m_{1} \neq m_{2}\right) \vee\left(m_{1}=m_{2}\right. \\
& \quad \wedge \text { DirectCorrelation }\left(\mathcal{D}, a_{1}, m_{1} . r e t\right) \wedge \operatorname{DirectCorrelation}\left(\mathcal{D}, m_{1} . \text { ret }, m_{1} \cdot p_{i}\right) \\
&\left.\wedge \text { DirectCorrelation }\left(\mathcal{D}, m_{1} \cdot p_{i}, a_{2}\right)\right)
\end{aligned}
$$

The predicate DirectCorrelation asserts if two variables are directly correlated according to Definition 1. These conditions state that there is a dependency between $m_{1}$ and $m_{2}$ through variable $x_{1}$, if the variable version $a_{1}$ is directly correlated with $a_{2}$ when $m_{1}$ and $m_{2}$ are two different atomic methods, or if the two methods $m_{1}$ and $m_{2}$ are the same, then we must be sure that the value of $x_{1}$ left out the scope of the method and then entered it again.

A process $p$ writes in a variable $x \in$ Vars if there is a write access on variable $x$ in one of the views of process $p$ :

$$
\operatorname{writes}(x, p) \Leftrightarrow \exists v \in V_{w}(p):(w, x) \in v
$$

The safety property for stale-value errors can be defined as the case where no process writes to a global variable that leaves, and then enters, the scope of an atomic method of another process. 
Table 1: Results for benchmarks - Set 1

\begin{tabular}{|c|c|c|c|c|c|c|c|c|c|c|}
\hline Tests & AV & \begin{tabular}{|c|c|c|} 
False \\
$M o T H$
\end{tabular} & $\begin{array}{l}\text { Nega } \\
\text { Artho }\end{array}$ & $\begin{array}{l}\text { ives } \\
\text { Teix. }\end{array}$ & $\mid \begin{array}{r}\text { Fals } \\
M o T H\end{array}$ & $\begin{array}{l}\text { Posit } \\
\text { Artho }\end{array}$ & $\begin{array}{l}\text { ives } \\
\text { Teix. }\end{array}$ & $\mid \begin{array}{l}\text { Acc. } \\
\text { Vars }\end{array}$ & LOC $\mid$ & $\begin{array}{l}\text { Time } \\
\text { (sec.) }\end{array}$ \\
\hline Connection [4] & 2 & 0 & 1 & 1 & 0 & 0 & 1 & 34 & 112 & 45 \\
\hline Coord03 [2] & 1 & 0 & 0 & 0 & 0 & 0 & 3 & 13 & 170 & 43 \\
\hline Local [2] & 1 & 0 & 1 & 0 & 0 & 0 & 1 & 3 & 33 & 42 \\
\hline NASA [2] & 1 & 0 & 0 & 0 & 0 & 0 & 0 & 7 & 121 & 43 \\
\hline Coord04 [3] & 1 & 0 & 0 & 0 & 0 & 0 & 3 & 7 & 47 & 40 \\
\hline Buffer [3] & 0 & 0 & 0 & 0 & 1 & 0 & 7 & 8 & 64 & 41 \\
\hline DoubleCheck [3] & 0 & 0 & 0 & 0 & 1 & 0 & 2 & 7 & 51 & 41 \\
\hline StringBuffer [5] & 1 & 0 & 1 & 1 & 0 & 0 & 0 & 12 & 52 & 44 \\
\hline Account [9] & 1 & 0 & 1 & 0 & 0 & 0 & 0 & 3 & 65 & 40 \\
\hline Jigsaw [9] & 1 & 0 & 0 & 0 & 0 & 0 & 1 & 33 & 145 & 40 \\
\hline OverReporting [9] & 0 & 0 & 0 & 0 & 0 & 0 & 2 & 6 & 52 & 42 \\
\hline UnderReporting [9] & 1 & 0 & 1 & 0 & 0 & 0 & 0 & 3 & 31 & 39 \\
\hline Allocate Vector [6] & 1 & 0 & 1 & 0 & 0 & 0 & 1 & 24 & 304 & 41 \\
\hline Knight [7] & 1 & 0 & 1 & 0 & 0 & 0 & 2 & 10 & 223 & 41 \\
\hline Arithmetic Database [7] & 3 & 0 & 3 & 1 & 1 & 0 & 0 & 24 & 416 & 54 \\
\hline Total & 15 & $\mathbf{0}$ & 10 & 3 & 3 & $\mathbf{0}$ & 23 & - & - & - \\
\hline
\end{tabular}

\section{Definition 11 (Stale-Value Safety).}

$$
\forall p \in \mathcal{P}_{\mathcal{S}},\left(m_{1}, x, m_{2}\right) \in \mathcal{D}_{\mathcal{V}}: \neg \text { writes }(x, p) \text { where } \mathcal{P}_{\mathcal{S}} \text { is the set of processes }
$$

If there is a view dependency for variable $x$ and there is a process $p$ that writes on that variable then a stale-value error is detected.

\section{Evaluation}

To evaluate the accuracy of our algorithms and techniques, we adapted and implemented the theoretical framework described in the previous sections to the Java Bytecode language, where the atomic methods are defined using the @Atomic method annotation. We used the data-flow analysis infrastructure of the Soot framework [8] to implement all the described analysis.

Our tool starts by parsing a Java bytecode program and computing a set of analysis, namely: process analysis to identify which threads may exists when executing the program; instance type analysis to handle Java interfaces and dynamic dispatching; views analysis, to compute the views of each atomic method; inter-procedural causal dependency analysis, to compute dependencies between variables used in assignments and conditional code blocks. Once all these analysis are concluded, the tool creates the causal dependency graph. Another analysis is then ran over this dependency graph to identify atomic blocks that break the atomicity violation safety properties.

Besides comparing our results with those reported on the literature for individual benchmarks, we did an exhaustive comparison with two other approaches: Artho et al's [2], because our approach is an extension of this work; and Teixeira's [7], because their results are currently a reference for the field. The results 
Table 2: Results for benchmarks - Set 2

\begin{tabular}{|c|c|c|c|c|c|c|c|c|}
\hline Tests & AV & $\begin{array}{l}\text { False } \\
\text { Ours }\end{array}$ & $\begin{array}{c}\text { Negatives } \\
\text { Artho }\end{array}$ & $\begin{array}{l}\text { False } \\
\text { Ours }\end{array}$ & $\begin{array}{c}\text { Positives } \\
\text { Artho }\end{array}$ & $\begin{array}{l}\text { Acc. } \\
\text { Vars }\end{array}$ & LOC & $\begin{array}{l}\text { Time } \\
\text { (sec.) }\end{array}$ \\
\hline Elevator [9] & 16 & 0 & 16 & 6 & 4 & 39 & 558 & 46 \\
\hline Philo [9] & 0 & 0 & 0 & 2 & 0 & $9 / 594$ & 96 & $45 / 612$ \\
\hline Tsp [9] & 0 & 0 & 0 & 2 & 0 & 635 & 795 & 869 \\
\hline Store & 2 & 0 & 1 & 0 & 1 & $44 / 608$ & 901 & $149 / 1763$ \\
\hline Total & 18 & $\mathbf{0}$ & 17 & 10 & 5 & - & - & - \\
\hline
\end{tabular}

presented were obtained by running our tool with the algorithms described in this paper; by using Artho et al's algorithm implemented with static analysis techniques (rather than the dynamic analysis reported in [2]); and by running Teixeira's tool on the Java source (instead of the Bytecode).

Tables 1 and 2 summarize the results achieved by applying our tool to a set of benchmarking programs, most of them well known from related works and compares them with the two works cited above. Teixeira's tool was unable to process some of the benchmarks, so they are reported in a separate second set. Columns $A V$ indicate the number of known atomicity violations, false negatives indicate the number of known program atomicity violations that were missed by the approach ${ }^{2}$, false positives indicate the number of reported but non-existing atomicity violations, Acc. Vars indicate the number of variables accessed inside atomic regions and is an indication of the problem size, together with the number of $L O C$, and how long it took for our analysis to run.

In the case of Table 2, the benchmarks Philo and Store have two different values for accessed variables and time. The second values report on the original benchmarks, which includes some (non-essential) calls to I/O methods in the JDK library. The first values report on a tailored version of the benchmarks where those calls to the JDK library were commented.

For the benchmarks listed in Table 1, our approach revealed a very high accuracy by reporting no false negatives and only three false positives. The false positive in the Buffer benchmark is due to an assumption claim from its authors that is not implemented in the actual code. The information collected by the Causal Dependency Analysis is incomplete and imprecise and originates false positives in the Double Check and Arithmetic Database benchmarks while checking for stale-value errors, which are not detected by Artho et al's approach.

For the benchmarks listed in Table 2, our appropriate again reveled very high accuracy, as although it reported 10 false positives (vs. only 5 from Artho et al's), it reported zero false negatives (vs. 17 from Artho et al's). These benchmarks also indicate that our algorithms scale well with the the size of the problem, both in the number of accessed variables inside the atomic blocks and the number of lines of code.

\footnotetext{
${ }^{2}$ The identification of false negative is only possible because the sets of atomicity violations in the benchmarking programs are well known.
} 


\section{Conclusions}

In this paper we presented a novel approach to detect high-level dataraces and stale-value errors in concurrent programs. The proposed approach relies on the notion of causal dependencies to improve the precision of previous detection techniques. The high-level dataraces are detected using an algorithm based on a previous work by Artho et al. refined to distinguish between read and write accesses and extended with the information given by the causal dependencies. The stale-value errors are detected using the information given by the causal dependencies, which exposes the values of variables that escaped an atomic block and entered into another atomic block.

Our detection analysis still remains unsound mainly due to the absence of pointer analysis and to the way that views are computed. But these design decisions allowed us to maintain the scalability of our approach without incurring in a strong precision loss, as our experimental results confirm.

We evaluated our analysis techniques with well known examples from the literature and compared them to previous works. Our results show that we are able to detect all atomicity violations present in the examples, while reporting a low number of false positives.

\section{References}

1. Alpern, B., Wegman, M.N., Zadeck, F.K.: Detecting equality of variables in programs. In: Proc. of the 15th ACM SIGPLAN-SIGACT symposium on Principles of programming languages. pp. 1-11. POPL '88, ACM, New York, NY, USA (1988)

2. Artho, C., Havelund, K., Biere, A.: High-level data races. Software Testing, Verification and Reliability 13(4), 207-227 (Dec 2003)

3. Artho, C., Havelund, K., Biere, A.: Using block-local atomicity to detect stale-value concurrency errors. In: ATVA. pp. 150-164 (2004)

4. Beckman, N.E., Bierhoff, K., Aldrich, J.: Verifying correct usage of atomic blocks and typestate. SIGPLAN Not. 43(10), 227-244 (2008)

5. Flanagan, C., Freund, S.N.: Atomizer: a dynamic atomicity checker for multithreaded programs. In: POPL '04: Proceedings of the 31st ACM SIGPLANSIGACT symposium on Principles of programming languages. pp. 256-267. ACM, New York, NY, USA (2004)

6. IBM HRL - Concurrency Testing Repository

7. Teixeira, B., Lourenço, J.M., Farchi, E., Dias, R.J., Sousa, D.G.: Detection of transactional memory anomalies using static analysis. In: Proceedings of the 8th Workshop on Parallel and Distributed Systems: Testing, Analysis, and Debugging. pp. 26-36. PADTAD '10, ACM, New York, NY, USA (2010)

8. Vallée-Rai, R., Hendren, L., Sundaresan, V., Lam, P., Gagnon, E., Co, P.: Soot - a Java optimization framework. In: Proceedings of CASCON 1999. pp. 125-135 (1999), http://www.sable.mcgill.ca/publications

9. von Praun, C., Gross, T.R.: Static detection of atomicity violations in objectoriented programs. In: Journal of Object Technology. p. 2004 (2003)

10. Wang, L., Stoller, S.: Run-Time Analysis for Atomicity. Electronic Notes in Theoretical Computer Science 89(2), 191-209 (Oct 2003) 